\title{
Performance analysis of a single-server ATM queue with a priority scheduling
}

\author{
Joris Walraevens*, Bart Steyaert and Herwig Bruneel \\ SMACS Research Group \\ Ghent University, Vakgroep TELIN (TW07) \\ Sint-Pietersnieuwstraat 41, B-9000 Gent, Belgium.
}

Phone: 0032-9-2648902

Fax: 0032-9-2644295

E-mail: \{jw,bs,hb\}@telin.rug.ac.be

\begin{abstract}
Scope and purpose
Queueing theory is an important subject in computers and operations research. Buffers/queues are used, e.g. in telecommunication networks, to store information that cannot be transmitted instantaneously. The study of the buffer behavior is important since network performance is directly related to it. Queues with a priority scheduling discipline are an important subject in queueing theory. As a result, these type of queues are thoroughly studied in the past, especially in continuous time. In discrete-time queueing models on the other hand, this type of queues is not as widely studied. Discrete-time queueing models are suitable for the performance evaluation of Asynchronous Transfer Mode (ATM) switches. In ATM, different types of traffic need different Quality of Service (QoS) standards. The delay characteristics of delay-sensitive traffic (e.g., voice) are more stringent than those of delay-insensitive traffic (e.g., data). We can thus give priority to delay-sensitive traffic over delay-insensitive traffic, thus trying to reduce the
\end{abstract}

* Corresponding author 
delay of the delay-sensitive traffic. This paper studies the impact of a priority scheduling on the buffer characteristics.

\begin{abstract}
In this paper, we consider a discrete-time queueing system with head-of-line (HOL) priority. First, we will give some general results on a GI-1-1 queue with priority scheduling. In particular, we will derive expressions for the Probability Generating Function of the system contents and the cell delay. Some performance measures (such as mean, variance and approximate tail distributions) of these quantities will be derived, and used to illustrate the impact and significance of priority scheduling in an ATM output queueing switch.
\end{abstract}

\title{
Key words
}

Discrete-time queueing models, priority scheduling, ATM switch 


\section{Introduction}

In recent years, there has been much interest in ATM as a promising technology for transport of high-bandwidth applications. Especially its well-defined QoS guarantee makes it extremely suitable for multimedia applications. Different types of traffic need different QoS standards. For real-time applications, it is important that mean delay and delay-jitter are not too large, while for non real-time applications, the Cell Loss Ratio (CLR) is the restrictive quantity.

In general, one can distinguish two priority categories, which will be referred to as Delay priority and Loss priority. Delay priority scheduling tries to reduce the delay of delay-sensitive traffic (such as voice). This is done by using a more sophisticated type of scheduling than the simple FIFO scheduling. Priority is given to delay-sensitive traffic over delay-insensitive traffic. Several types of Delay priority (or cell scheduling) schemes (such as Weighted-Round-Robin (WRR), WeightedFair-Queueing(WFQ)) have been proposed and analyzed for ATM applications, each with their own specific algorithmic and computational complexity (see e.g. [11] and the references therein). On the other hand, Loss priority schemes attempt to reduce the cell loss of loss-sensitive traffic (such as data). Again, various types of Loss priority (or cell discarding) strategies for ATM (such as Push-Out Buffer (POB), Partial Buffer Sharing (PBS)) have been presented in the literature (see e.g. [19]). An overview of both types of priority can be found in [1].

In this paper, we will focus on the effect of HOL (or non-preemptive) Delay priority scheduling. We assume that delay-sensitive traffic has absolute priority over delay-insensitive traffic, i.e., when a server becomes idle, a cell of delay-sensitive traffic, when available, will always be scheduled next. This is the most drastic type of Delay priority scheduling, but also the easiest one to implement. In the existing literature, there have been a number of contributions with respect to this priority scheme. In [10,12-18], HOL priority queues have been analyzed with a wide variety of arrival and service time distributions.

In this paper, we use an analysis based on generating functions for assessing the performance of ATM buffers with a priority scheduling discipline. From these generating functions, we can then easily calculate expressions for some interesting performance measures, such as mean value, 
variance and approximations for the tail distribution of the buffer contents and cell delay. These closed-form expressions require virtually no computational effort at all, and are well-suited for evaluating the impact of the various system parameters on the overall performance. We will also show that our results can be applied to the case of an ATM output-queueing switch with HOL priority scheduling. There have been a number of contributions with respect to switches with output queueing, in the case of a single traffic type and a FIFO scheduling discipline, such as $[4,5,9]$.

The contribution of this paper concerns the model that is considered, the solution technique that is used, as well as the results that are generated. First, as far as the model is concerned, the main difference with the articles involved with HOL priority queues listed above is that, for the case of a multiclass output-queueing switch, the arrival processes of the different types of cells are not mutually independent. Therefore the different classes can not be analyzed separately (i.e., as a model with server interruptions for low priority cells as demonstrated in section 5), which complicates the analysis. Secondly, we want to show that a generating-functions solution method is extremely suitable for analyzing this type of buffers with a priority scheduling discipline, whereas existing models are mainly based on matrix-analytic methods. Finally, determining the tail behavior of the buffer contents and cell delay is one of the main contributions of the paper. Although these are important quantities in the evaluation of QoS of high- and low-priority cell streams, this has received only few attention up to now. We will also show that the distribution of the buffer contents and cell delay of low priority cells not necessarily has a geometric asymptotic behavior.

The outline is as follows. First, we consider a single queue with a general arrival distribution. In the following section, we will introduce the mathematical model. In section 3 and 4 we will analyze the steady-state system contents and cell delay. In section 5 , we discuss the results derived in the former sections and we calculate the moments of the system contents and cell delay in section 6. We study the tail behavior of the system contents and cell delay in section 7 . We apply the obtained results to an output queueing switch with Bernoulli arrivals, and discuss the impact of a 
HOL priority scheduling discipline in section 8 . Some conclusions are formulated in section 9 .

\section{Mathematical model}

We consider a discrete-time single-server queueing system with infinite buffer space. Time is assumed to be slotted, where 1 slot equals the transmission time of a cell. There are 2 types of traffic arriving in the system, namely cells of class- 1 and cells of class- 2 . We denote the number of arrivals of class- $j$ during slot $k$ by $a_{j, k}(j=1,2)$. Both types of cell arrivals are assumed to be i.i.d. from slot-to-slot and are characterized by the joint probability mass function (pmf) $a(m, n)$,

$$
a(m, n) \triangleq \operatorname{Prob}\left[a_{1, k}=m, a_{2, k}=n\right]
$$

and joint probability generating function (pgf) $A\left(z_{1}, z_{2}\right)$,

$$
A\left(z_{1}, z_{2}\right) \triangleq E\left[z_{1}^{a_{1, k}} z_{2}^{a_{2, k}}\right]
$$

Notice that the number of cell arrivals from different classes (within a slot) can be correlated. Further, we denote the total number of arriving cells during slot $k$ by $a_{T, k} \triangleq a_{1, k}+a_{2, k}$ and its pgf is defined as $A_{T}(z) \triangleq E\left[z^{a_{T, k}}\right]=A(z, z)$. In the same way, we define the marginal pgf's of the number of arrivals from class- 1 and class- 2 during a slot by $A_{1}(z) \triangleq E\left[z^{a_{1, k}}\right]=A(z, 1)$ and $A_{2}(z) \triangleq E\left[z^{a_{2, k}}\right]=A(1, z)$ respectively. We furthermore denote the arrival rate of class- $j(j=1,2)$ by $\lambda_{j}=A_{j}^{\prime}(1)$ and the total arrival rate by $\lambda_{T}=A_{T}^{\prime}(1)=A_{1}^{\prime}(1)+A_{2}^{\prime}(1)$. The system has one server that provides the transmission of cells, at a rate of 1 cell per slot. We assume a stable system, i.e., $\lambda_{T}<1$.

Newly arriving cells can enter service at the beginning of the slot following their arrival slot at the earliest. Class- 1 cells are assumed to have priority over class- 2 cells, and within one class the service discipline is FCFS. Due to the priority scheduling mechanism, it is as if class- 1 cells are stored in front of class- 2 cells in the queue. So, if there are any class- 1 cells in the queue at the beginning of a slot, the one with the longest waiting time will be served next. If, on the other 
hand, no class- 1 cells are present in the queue at the beginning of a particular slot, the class- 2 cell with the longest waiting time, if any, will be served.

\section{System contents}

In this section, we concentrate on the effect of the HOL priority scheduling discipline on the probability generating function of the steady-state system contents, which represents the number of cells in the buffer. This was already done in [13] - for a more general queueing system - but it is useful to give the analysis in our special case. We denote the system contents of class- $j$ at the beginning of slot $k$ by $u_{j, k}(j=1,2)$ and the total system contents at the beginning of slot $k$ by $u_{T, k}$. Furthermore, we denote the joint pgf of $u_{1, k}$ and $u_{2, k}$ by $U_{k}\left(z_{1}, z_{2}\right)$, i.e.,

$$
U_{k}\left(z_{1}, z_{2}\right) \triangleq E\left[z_{1}^{u_{1, k}} z_{2}^{u_{2, k}}\right]
$$

The system contents of both types of cells is characterized by the following system equations:

$$
\begin{aligned}
& u_{1, k+1}=\left[u_{1, k}-1\right]^{+}+a_{1, k} ; \\
& u_{2, k+1}= \begin{cases}{\left[u_{2, k}-1\right]^{+}+a_{2, k}} & \text { if } u_{1, k}=0 \\
u_{2, k}+a_{2, k} & \text { if } u_{1, k}>0\end{cases}
\end{aligned}
$$

where $[.]^{+}$denotes the maximum of the argument and 0 . The first equation follows from the observation that class-1 cells are not influenced by class- 2 cells. A class- 2 cell on the other hand can only be served, if there are no class-1 cells in the system. This leads to the second equation. Using these system equations, we can form the following relation between $U_{k+1}\left(z_{1}, z_{2}\right)$ and $U_{k}\left(z_{1}, z_{2}\right)$

$$
U_{k+1}\left(z_{1}, z_{2}\right)=A\left(z_{1}, z_{2}\right) \frac{z_{2} U_{k}\left(z_{1}, z_{2}\right)+\left(z_{1}-z_{2}\right) U_{k}\left(0, z_{2}\right)+z_{1}\left(z_{2}-1\right) U_{k}(0,0)}{z_{1} z_{2}}
$$


Since we are interested in the steady-state distribution of the system contents, we define $U\left(z_{1}, z_{2}\right)$

as

$$
U\left(z_{1}, z_{2}\right) \triangleq \lim _{k \rightarrow \infty} U_{k}\left(z_{1}, z_{2}\right)
$$

Applying this limit in equation (1), we find the following expression for $U\left(z_{1}, z_{2}\right)$,

$$
U\left(z_{1}, z_{2}\right)=A\left(z_{1}, z_{2}\right) \frac{\left(z_{1}-z_{2}\right) U\left(0, z_{2}\right)+z_{1}\left(z_{2}-1\right) U(0,0)}{z_{2}\left(z_{1}-A\left(z_{1}, z_{2}\right)\right)}
$$

There are two quantities yet to be determined in the right hand side of equation (2), namely the function $U\left(0, z_{2}\right)$ and the constant $U(0,0)$. Applying Rouché's theorem, it can be proven that for a given value of $z_{2}\left(\left|z_{2}\right| \leq 1\right)$, the equation $z_{1}=A\left(z_{1}, z_{2}\right)$ has one solution in the unit circle for $z_{1}$, which will be denoted by $Y\left(z_{2}\right)$ in the remainder, and which is implicitly defined by $Y(z) \triangleq A(Y(z), z)$. Since $Y\left(z_{2}\right)$ is a zero of the denominator of the right hand side of (2) and since a generating function remains finite in the unit circle, $Y\left(z_{2}\right)$ must be a zero of the numerator too. We thus find

$$
U\left(0, z_{2}\right)=U(0,0) \frac{Y\left(z_{2}\right)\left(z_{2}-1\right)}{z_{2}-Y\left(z_{2}\right)}
$$

Substituting this result in equation (2) yields

$$
U\left(z_{1}, z_{2}\right)=U(0,0) \frac{A\left(z_{1}, z_{2}\right)\left(z_{2}-1\right)}{z_{2}-Y\left(z_{2}\right)} \frac{z_{1}-Y\left(z_{2}\right)}{z_{1}-A\left(z_{1}, z_{2}\right)}
$$

$U(0,0)$ can be found by applying the normalization condition $U(1,1)=1$. Using de l' Hopital's rule gives the expected result for the probability of having an empty system: $U(0,0)=1-\lambda_{T}$. From equation (3), we easily obtain an expression for the pgf $U_{T}(z)$ describing the total system contents

$$
\begin{aligned}
U_{T}(z) & \triangleq \lim _{k \rightarrow \infty} \mathrm{E}\left[z^{u_{T, k}}\right]=U(z, z) \\
& =\left(1-\lambda_{T}\right) \frac{A_{T}(z)(z-1)}{z-A_{T}(z)}
\end{aligned}
$$


We can also calculate the pgf $U_{j}(z)(j=1,2)$ of the system contents of class- $j$, namely

$$
\begin{aligned}
U_{1}(z) & \triangleq \lim _{k \rightarrow \infty} \mathrm{E}\left[z^{u_{1, k}}\right]=U(z, 1) \\
& =\left(1-\lambda_{1}\right) \frac{A_{1}(z)(z-1)}{z-A_{1}(z)}, \\
U_{2}(z) & \triangleq \lim _{k \rightarrow \infty} \mathrm{E}\left[z^{u_{2, k}}\right]=U(1, z) \\
& =\left(1-\lambda_{T}\right) \frac{A_{2}(z)(z-1)}{z-Y(z)} \frac{1-Y(z)}{1-A_{2}(z)} .
\end{aligned}
$$

We will discuss these results in section 5 .

\section{Cell delay}

The cell delay is defined as the total amount of time that a cell spends in the system, i.e., the number of slots between the end of the cell's arrival slot and the end of its departure slot. In this section, we will derive expressions for the pgf's of the cell delay of both classes.

We can analyze the cell delay of class- 1 cells as if they are the only type of cells in the system. This is e.g. done in [3] and the pgf of the cell delay of class- 1 cells is given by

$$
D_{1}(z)=\frac{1-\lambda_{1}}{\lambda_{1}} \frac{z\left(A_{1}(z)-1\right)}{z-A_{1}(z)}
$$

The analysis of the cell delay of a class- 2 cell is more complicated. Consider a logical equivalent queueing system where all high priority cells are stored in front of the class- 2 cells, and let us tag an arbitrary class- 2 cell that arrives in the system. The amount of time it spends in the system equals

$$
d_{2}=\sum_{j=1}^{\left[u_{T, k}-1\right]^{+}+f_{2, k}} v_{j}^{0}+1,
$$

where slot $k$ is assumed to be the arrival slot of the tagged cell, $f_{2, k}$ is defined as the total number of cells that arrive during the arrival slot of the tagged cell, but which have to be served before it, and $v_{j}^{0}$ represents the number of slots it takes for the tagged cell to move one position ahead 
in the queue, e.g., from position $j$ to position $j-1$ (see Figure 1). In case of FIFO scheduling, $v_{j}^{0}$ would equal 1. For HOL priority scheduling, this is not necessarily the case, since new class-1 cells can arrive while the tagged cell is waiting in the queue and these class- 1 cells have to be served before the tagged cell. More specific, assume that the tagged cell is stored in the $j$-th position in the queue at the beginning of the $l$-th slot $\left(0<j \leq\left[u_{T, k}-1\right]^{+}+f_{2, k}\right)$. If no class- 1 cells arrive during slot $l, v_{j}^{0}$ equals 1 . If $a_{1, l}(>0)$ class-1 cells arrive during this slot on the other hand, the tagged cell will move back to position $j+a_{1, l}-1$ in the queue at the beginning of slot $l+1$, since these class- 1 cells have to be served before all class- 2 cells, and thus before the tagged one (Figure 1). If we then define $v_{j, i}^{1}\left(j \leq i \leq j+a_{1, l}-1\right)$ as the number of slots it takes the tagged cell to go from position $i$ to position $i-1$, it is clear that $v_{j}^{0}$ can be calculated as follows,

$$
v_{j}^{0}=\sum_{i=0}^{a_{1, l}-1} v_{j, j+i}^{1}+1 .
$$

[Figure 1 about here.]

Now, one can easily see that all $v_{j}^{0}$ and $v_{j, i}^{1}$ form a set of mutually independent random variables since they depend on the number of class-1 cell arrivals during different slots. From a stochastic point-of-view, these are i.i.d. variables and, as a result, are characterized by the same pgf $V(z)$. From equation (9), it can be seen that $V(z)$ satisfies

$$
V(z)=z A_{1}(V(z))
$$

Furthermore, $f_{2, k}$ is the sum of all the class- 1 cells that arrive during the same slot as the tagged one, and of the class- 2 cells that have arrived before it during its arrival slot. The pgf of $f_{2, k}$ can be calculated taking into account that an arbitrary tagged cell is more likely to arrive in a larger bulk (e.g. [3]), yielding

$$
F_{2}(z)=\frac{A_{T}(z)-A_{1}(z)}{\lambda_{2}(z-1)} .
$$

Using equations (4) and (11) in the $z$-transform of equation (8) eventually gives us the steady-state 
pgf of $d_{2}$, i.e.,

$$
D_{2}(z)=\frac{1-\lambda_{T}}{\lambda_{2}} \frac{z\left(A_{T}(V(z))-A_{1}(V(z))\right)}{V(z)-A_{T}(V(z))},
$$

where $V(z)$ is implicitly determined by equation (10).

\section{Discussion of the results and special relations}

In this section, we will discuss some of the results from the former sections. First, we notice that the pgf of the total system contents (equation (4)) is the same as for a single class system with an identical cell arrival process described by $A_{T}(z)$. Indeed, since the service time is deterministic and equal to 1 slot for the two classes, the scheduling has no impact on the total system contents.

Second, we see that the system contents of class-1 cells (equation (5)) is not influenced by class-2 cells and furthermore that its pgf has the same structure as $U_{T}(z)$. This is of course due to the HOL priority scheduling. For class- 1 cells, it seems as if no class- 2 cells are present in the system. Consequently, since the scheduling is FIFO within class-1, $U_{1}(z)$ and $D_{1}(z)$ fulfill the following relation (see [20]):

$$
U_{1}(z)=1-\lambda_{1}+\lambda_{1} D_{1}(z)
$$

It is easily verified that indeed (5) and (7) satisfy this equation.

In the special case that the number of arrivals of class- 1 and class- 2 cells are uncorrelated, i.e. $A\left(z_{1}, z_{2}\right)=A_{1}\left(z_{1}\right) A_{2}\left(z_{2}\right)$, we can calculate the system contents of class-2 cells in an alternative way. Since class- 2 cells can only be served when there are no class- 1 cells in the system, we can model the system, with respect to class- 2 cells, in terms of a system with server interruptions. The server is blocked for class- 2 cells if there are class- 1 cells waiting to be sent, and it is available if there are none. We can then calculate the pgf of the duration of busy and idle period of class- 1 cells, i.e., the time period during which there are class- 1 cells in the system (i.e., $u_{1}>0$ ) and the time period during which there are no such cells (i.e., $u_{1}=0$ ), respectively. It is easily verified 
that the duration of the idle period is geometrically distributed, i.e., its pgf is given by

$$
I(z)=\frac{\left(1-A_{1}(0)\right) z}{1-A_{1}(0) z}
$$

The calculation of the busy period is a bit more involved, and can be found in [3] for a general service time distribution. In case of deterministic service times of one slot, it is implicitly given by the following formula:

$$
B(z)=\frac{A_{1}\left(z\left(\left(1-A_{1}(0)\right) B(z)+A_{1}(0)\right)\right)-A_{1}(0)}{1-A_{1}(0)} .
$$

Note that the lengths of consecutive busy and idle periods are statistically independent. It is clear that when the system is busy with respect to class- 1 cells, it is blocked for class- 2 cells. Therefore, with respect to class-2 cells, the system can be modelled as a single-server buffer with server interruptions, for which the lengths of consecutive available and blocked periods are i.i.d. and their respective pgf's are given by equation (13) and (14). Such a queueing system has already been analyzed in [2]. Translating the results from this analysis to our case, the pgf of the system contents of class- 2 cells becomes

$$
U_{2}(z)=\left(1-\lambda_{T}\right) \frac{A_{2}(z)(z-1)}{z-X(z)} \frac{1-X(z)}{1-A_{2}(z)}
$$

with

$$
X(z)=A_{1}(X(z)) A_{2}(z)
$$

Equations (6) and (15) lead to the same result for $U_{2}(z)$, when $X(z)=Y(z)$. This is the case when the number of class- 1 and class- 2 arrivals during a slot are uncorrelated. 


\section{Calculation of moments}

The functions $Y(z)$ and $V(z)$, defined in sections 3 and 4, can only be explicitly found in case of some simple arrival processes. Their derivatives for $z=1$, necessary to calculate the moments of the system contents and the cell delay, on the contrary, can be calculated in closed-form. For example, the first derivatives are given by

$$
Y^{\prime}(1)=\frac{\lambda_{2}}{1-\lambda_{1}}, V^{\prime}(1)=\frac{1}{1-\lambda_{1}} .
$$

Let us define $\lambda_{i j}$ as

$$
\left.\lambda_{i j} \triangleq \frac{\partial^{2} A\left(z_{1}, z_{2}\right)}{\partial z_{i} \partial z_{j}}\right|_{z_{1}=z_{2}=1},
$$

with $i, j=1,2$. Now we can calculate the mean values of the system contents and cell delay of both classes by taking the first derivative of the respective pgf's for $z=1$. We find

$$
E\left[u_{T}\right]=\lambda_{T}+\frac{A_{T}^{\prime \prime}(1)}{2\left(1-\lambda_{T}\right)},
$$

for the mean value of total system contents,

$$
E\left[u_{1}\right]=\lambda_{1}+\frac{\lambda_{11}}{2\left(1-\lambda_{1}\right)},
$$

for the mean system contents of class- 1 cells and

$$
E\left[u_{2}\right]=\lambda_{2}+\frac{2 \lambda_{12}+\lambda_{22}}{2\left(1-\lambda_{T}\right)}+\frac{\lambda_{2} \lambda_{11}}{2\left(1-\lambda_{T}\right)\left(1-\lambda_{1}\right)},
$$

for the mean system contents of class-2 cells. It is easily verified that equations (16), (17) and (18) satisfy $E\left[u_{T}\right]=E\left[u_{1}\right]+E\left[u_{2}\right]$.

Furthermore, from equations (7) and (12), we derive the following expressions

$$
E\left[d_{1}\right]=1+\frac{\lambda_{11}}{2 \lambda_{1}\left(1-\lambda_{1}\right)},
$$


and

$$
E\left[d_{2}\right]=1+\frac{2 \lambda_{12}+\lambda_{22}}{2 \lambda_{2}\left(1-\lambda_{T}\right)}+\frac{\lambda_{11}}{2\left(1-\lambda_{T}\right)\left(1-\lambda_{1}\right)},
$$

for the mean cell delay of a class- 1 and a class- 2 cell respectively. We can see from equations (17)

- (20) that Little's law $E\left[u_{j}\right]=\lambda_{j} E\left[d_{j}\right](j=1,2)$ is fulfilled for both classes, as expected.

In a similar way, expressions for the variance of system contents and cell delay and some interesting correlation coefficients can be calculated by taking the appropriate derivatives of the respective generating functions as well. The expressions are nevertheless too exhaustive, but we will show them in some figures in section 8 .

\section{Tail behavior}

Not only the moments of the system contents and cell delay are important, but also, and especially, the tail distribution of these quantities, which are often used to impose statistical bounds on the guaranteed QoS for both classes.

From the generating functions of the total system contents, and of the system contents and cell delay of class- 1 and class- 2 cells derived in sections 3 and 4 , approximations of the tail probabilities can be derived using complex contour integration and residue theory. The procedure to find the corresponding probability mass function of a pgf, frequently used in the following of this section, is generally described in Appendix 1.

In order to determine the asymptotic behavior of the tail distribution, the dominant singularity of the respective generating functions is important. In e.g. [6] (wherein a single-class ATM queue with a FIFO scheduling discipline is analyzed), it is proven that the dominant singularity lies on the positive real axis and is larger than 1.

First we concentrate on the total system contents. Provided that the pgf $A_{T}(z)$ exhibits no long-tail behavior, which is assumed to be the case here, the dominant singularity $z_{T}$ of $U_{T}(z)$ is a zero of $z-A_{T}(z)$ and this singularity is a single pole. In the neighbourhood of this pole, we can 
approximate $U_{T}(z)$ by

$$
U_{T}(z) \approx \frac{K_{T}}{z_{T}-z},
$$

where $K_{T}$ can be found by substituting $z=z_{T}$ in (21). Using residue theory, the tail probability is easily found to yield

$$
\operatorname{Prob}\left[u_{T}=n\right] \approx\left(1-\lambda_{T}\right) \frac{z_{T}-1}{A_{T}^{\prime}\left(z_{T}\right)-1} z_{T}^{-n}
$$

for large enough $n$. The system contents of class-1 cells has an identical tail behavior:

$$
\operatorname{Prob}\left[u_{1}=n\right] \approx\left(1-\lambda_{1}\right) \frac{z_{H}-1}{A_{1}^{\prime}\left(z_{H}\right)-1} z_{H}^{-n}
$$

for large enough $n$, with $z_{H}$ the dominant singularity on the positive real axis of $U_{1}(z)$, i.e., $z_{H}$ is a zero of $z-A_{1}(z)$.

The tail behavior of the system contents of class- 2 cells is a bit more involved, since it is not a priori clear what the dominant singularity is of $U_{2}(z)$. This is due to the occurance of the function $Y(z)$ in (6), which is only implicitly defined. First we take a closer look at that function $Y(z)$. The first derivative of $Y(z)$ is given by

$$
Y^{\prime}(z)=\frac{A^{(2)}(Y(z), z)}{1-A^{(1)}(Y(z), z)}
$$

with $A^{(j)}\left(z_{1}, z_{2}\right) \triangleq \frac{\partial A\left(z_{1}, z_{2}\right)}{\partial z_{j}}(j=1,2)$. Consequently, $Y(z)$ has a singularity, denoted as $z_{B}$, where the denominator of $Y^{\prime}(z)$ becomes 0 , i.e., $A^{(1)}\left(Y\left(z_{B}\right), z_{B}\right)=1$. Since $Y(z)$ remains finite in the neighborhood of $z_{B}$, this singularity is not a simple pole. Applying the results from [7] one can show that in the neighbourhood of $z_{B}, Y(z)$ is approximately given by

$$
Y(z) \approx Y\left(z_{B}\right)-K_{Y} \sqrt{z_{B}-z}
$$

with $K_{Y}=\sqrt{\frac{2 A^{(2)}\left(Y\left(z_{B}\right), z_{B}\right)}{A^{(11)}\left(Y\left(z_{B}\right), z_{B}\right)}}$, which can be found by taking the limit $z \rightarrow z_{B}$ of $(25) . A^{(i j)}\left(z_{1}, z_{2}\right)$ is defined as $\frac{\partial^{2} A\left(z_{1}, z_{2}\right)}{\partial z_{i} \partial z_{j}}$ (for $\left.i, j=1,2\right)$. From equation (25) it becomes obvious that $z_{B}$ is a square- 
root branch point of $Y(z) . Y(z)$ has thus two real solutions when $z<z_{B}$ (the solution we are interested in is the one where $Y(z)<1$, if $z<1$ ), which coincide at $z_{B}$, and has no real solution when $z>z_{B} . z_{B}$ is then of course also a branch point of $U_{2}(z)$. A second potential singularity $z_{L}$ of $U_{2}(z)$ on the real axis is given by the positive zero of the denominator $z-Y(z)$, and is easily proven to be equal to $z_{T}$, if $z_{L}$ exists.

The tail behavior of the system contents of class- 2 cells is thus characterized by $z_{T}$ or $z_{B}$, depending on which is the dominant (i.e., smallest) singularity. Three types of tail behavior may thus occur, namely when $z_{L}=z_{T}<z_{B}, z_{L}=z_{T}=z_{B}$ and $z_{L}$ does not exist. In those three cases, $U_{2}(z)$ can be approximated in the neighbourhood of its dominant singularity by:

$$
U_{2}(z) \approx \begin{cases}\frac{K_{2}^{(1)}}{z_{T}-z} & \text { if } z_{L}=z_{T}<z_{B} \\ \frac{K_{2}^{(2)}}{\sqrt{z_{B}-z}} & \text { if } z_{L}=z_{T}=z_{B} \\ U_{2}\left(z_{B}\right)-K_{2}^{(3)} \sqrt{z_{B}-z} & \text { if } z_{L} \text { does not exist }\end{cases}
$$

where the constants $K_{2}^{(i)}(i=1,2,3)$ can be found by investigating the behavior of $U_{2}(z)$ in the neighborhood of this dominant singularity. Using residue theory, we find the tail probabilities for the three possible cases:

$$
\operatorname{Prob}\left[u_{2}=n\right] \approx\left\{\begin{array}{l}
\left(1-\lambda_{T}\right) \frac{A_{2}\left(z_{T}\right)\left(z_{T}-1\right)^{2}}{z_{T}\left(A_{2}\left(z_{T}\right)-1\right)\left(Y^{\prime}\left(z_{T}\right)-1\right)} z_{T}^{-n} \\
\frac{1-\lambda_{T}}{K_{Y}} \sqrt{\frac{1}{z_{B} \pi}} \frac{A_{2}\left(z_{B}\right)\left(z_{B}-1\right)^{2}}{A_{2}\left(z_{B}\right)-1} n^{-1 / 2} z_{B}^{-n} \\
\frac{\left(1-\lambda_{T}\right) K_{Y}}{2} \sqrt{\frac{z_{B}}{\pi}} \frac{A_{2}\left(z_{B}\right)\left(z_{B}-1\right)^{2}}{\left(A_{2}\left(z_{B}\right)-1\right)\left(z_{B}-Y\left(z_{B}\right)\right)^{2}} n^{-3 / 2} z_{B}^{-n}
\end{array}\right.
$$

for large enough $n$, if $z_{L}=z_{T}<z_{B}$, if $z_{L}=z_{T}=z_{B}$, and if $z_{L}$ does not exist respectively. The first expression constitutes a typical geometric tail behavior, the third expression is a typical non-geometric tail behavior and the second expression gives a transition between geometric and non-geometric tail behavior. The latter two expressions are found from the approximations of the generating functions by using the Theorem from Appendix 2 (which is a theorem stated in [8]). 
Let us now consider the cell delay. The dominant singularity of $D_{1}(z)$ is the same as the one of $U_{1}(z)$, and we can thus approximate the tail behavior of the delay of class- 1 cells by

$$
\operatorname{Prob}\left[d_{1}=n\right] \approx \frac{\left(1-\lambda_{1}\right)}{\lambda_{1}} \frac{z_{H}-1}{A_{1}^{\prime}\left(z_{H}\right)-1} z_{H}^{-n}
$$

for large enough $n$. The tail behavior of the delay of class-2 cells is again a bit more involved because of the appearance of the function $V(z)$ in $(12)$, which is only implicitly known. The first derivative of $V(z)$ is given by

$$
V^{\prime}(z)=\frac{A_{1}(V(z))}{1-z A_{1}^{\prime}(V(z))}
$$

which, similar as before, indicates that $V(z)$ also has a square root branch point $\hat{z}_{B}$, with $\hat{z}_{B} A_{1}^{\prime}\left(V\left(\hat{z}_{B}\right)\right)=$ 1. In the neighbourhood of $\hat{z}_{B}, V(z)$ is approximately given by

$$
V(z) \approx V\left(\hat{z}_{B}\right)-K_{V} \sqrt{\hat{z}_{B}-z}
$$

with $K_{V}=\sqrt{\frac{2 A_{1}\left(V\left(\hat{z}_{B}\right)\right)}{\hat{z}_{B} A_{1}^{\prime \prime}\left(V\left(\hat{z}_{B}\right)\right)}}$. A second singularity of $D_{2}(z)$ is given by the dominant zero $\hat{z}_{L}$ of $V(z)-A_{T}(V(z))$ on the real axis and is easily proven to equal $\frac{z_{T}}{A_{1}\left(z_{T}\right)}$, if $\hat{z}_{L}$ exists.

So, $D_{2}(z)$ can be approximated in the neighbourhood of his dominant singularity by:

$$
D_{2}(z) \approx \begin{cases}\frac{\hat{K}_{2}^{(1)}}{\frac{z_{T}}{A_{1}\left(z_{T}\right)}-z} & \text { if } \hat{z}_{L}=\frac{z_{T}}{A_{1}\left(z_{T}\right)}<\hat{z}_{B} \\ \frac{\hat{K}_{2}^{(2)}}{\sqrt{\hat{z}_{B}-z}} & \text { if } \hat{z}_{L}=\frac{z_{T}}{A_{1}\left(z_{T}\right)}=\hat{z}_{B} \\ D_{2}\left(\hat{z}_{B}\right)-\hat{K}_{2}^{(3)} \sqrt{\hat{z}_{B}-z} & \text { if } \hat{z}_{L} \text { does not exist }\end{cases}
$$

where the constants $\hat{K}_{2}^{(i)}(i=1,2,3)$ can be found by investigating $D_{2}(z)$ in the neighborhood of its dominant singularity. By using residue theory once again, the asymptotic behavior of $D_{2}(z)$ is 
given by

$$
\operatorname{Prob}\left[d_{2}=n\right] \approx\left\{\begin{array}{l}
\frac{1-\lambda_{T}}{\lambda_{2}} \frac{\left(z_{T}-A_{1}\left(z_{T}\right)\right)\left(A_{1}\left(z_{T}\right)-z_{T} A_{1}^{\prime}\left(z_{T}\right)\right)}{\left(A_{1}\left(z_{T}\right)\right)^{2}\left(A_{T}^{\prime}\left(z_{T}\right)-1\right)}\left(\frac{z_{T}}{A_{1}\left(z_{T}\right)}\right)^{-n} \\
\frac{1-\lambda_{T}}{\lambda_{2} K_{V} \sqrt{\hat{z}_{B} \pi}} \frac{\hat{z}_{B} A_{T}\left(V\left(\hat{z}_{B}\right)\right)-V\left(\hat{z}_{B}\right)}{A_{T}^{\prime}\left(V\left(\hat{z}_{B}\right)\right)-1} n^{-1 / 2} \hat{z}_{B}^{-n} \\
\frac{\left(1-\lambda_{T}\right) K_{V}}{2 \lambda_{2} \sqrt{\pi / \hat{z}_{B}}} \frac{\left(\hat{z}_{B}-1\right)\left(V\left(\hat{z}_{B}\right) A_{T}^{\prime}\left(V\left(\hat{z}_{B}\right)\right)-A_{T}\left(V\left(\hat{z}_{B}\right)\right)\right)}{\left(V\left(\hat{z}_{B}\right)-A_{T}\left(V\left(\hat{z}_{B}\right)\right)\right)^{2}} n^{-3 / 2} \hat{z}_{B}^{-n}
\end{array}\right.
$$

if $\hat{z}_{L}=\frac{z_{T}}{A_{1}\left(z_{T}\right)}<\hat{z}_{B}$, if $\hat{z}_{L}=\frac{z_{T}}{A_{1}\left(z_{T}\right)}=\hat{z}_{B}$, and if $\hat{z}_{L}$ does not exist respectively. The first expression has a typical geometric tail behavior, the third expression has a typical non-geometric tail behavior and the second expression gives a transition between geometric and non-geometric tail behavior.

A quantity of practical interest is the probability that a cell has a delay that exceeds a bound $D$. We find

$$
\operatorname{Prob}\left[d_{1}>D\right] \approx \frac{\operatorname{Prob}\left[d_{1}=D+1\right] \hat{z}_{H}}{\hat{z}_{H}-1}
$$

for the probability that the delay of a class- 1 cell is larger than a bound $D$. This can be found by summing equation (27) for all appropriate values of $n$. Analogously, we can calculate the probability that a class-2 cell exceeds a bound D by summing equation (30) for the appropriate values of $n$. We find

$$
\operatorname{Prob}\left[d_{2}>D\right] \approx \begin{cases}\frac{\operatorname{Prob}\left[d_{2}=D+1\right] \hat{z}_{L}}{\hat{z}_{L}-1} & \text { if } \hat{z}_{L}=\frac{z_{T}}{A_{1}\left(z_{T}\right)}<\hat{z}_{B} \\ \frac{\operatorname{Prob}\left[d_{2}=D+1\right] \hat{z}_{B}}{\hat{z}_{B}-1} & \text { if } \hat{z}_{L}=\frac{z_{T}}{A_{1}\left(z_{T}\right)}=\hat{z}_{B} \\ \frac{\operatorname{Prob}\left[d_{2}=D+1\right] \hat{z}_{B}}{\hat{z}_{B}-1} & \text { if } \hat{z}_{L} \text { does not exist }\end{cases}
$$

where we used the approximation that $\sum_{n=D+1}^{\infty} n^{-a} z^{-n} \approx(D+1)^{-a} \sum_{n=D+1}^{\infty} z^{-n}$, with $a=1 / 2$ or $3 / 2$ and which holds for large enough $D$. Some similar expressions can be found for the probability that the system contents exceeds a certain bound. 
Since the results obtained in this section are approximate (due to the dominant pole approximative method), the question remains if the expressions are accurate. From the analysis in [6], it follows that the approximation of the tail probabilities, obtained through the dominant pole method, are better when the dominant pole is more dominant (i.e., the higher the moduli of the other poles, the better the quality of the approximation) and when we go further in the tail of the distribution (i.e., when coefficient $n$ in (22)-(23), (26)-(27) and (30) is higher). We will show in section 8 that the approximate results for the tail probabilities obtained in this section are satisfactory.

\section{Application}

In this section, we apply our results from the former sections to an ATM output-queueing switch. We consider a non-blocking output-queueing switch with $N$ inlets and $N$ outlets (Figure 2). We assume two types of traffic. Traffic of class-1 is delay-sensitive (for instance voice) and traffic of class-2 is assumed to be delay-insensitive (for instance data). We investigate the effect of HOL priority scheduling, as presented in the former of this paper.

[Figure 2 about here.]

The cell arrivals on each inlet are assumed to be i.i.d., and generated by a Bernoulli process with arrival rate $\lambda_{T}$. An arriving cell is assumed to be of class- $j$ with probability $\lambda_{j} / \lambda_{T}(j=1,2)$ $\left(\lambda_{1}+\lambda_{2}=\lambda_{T}\right)$. The incoming cells are then routed to the output queue corresponding to their destination, in an independent and uniform way. Therefore, the output queues behave identically and we can concentrate on the analysis of 1 output queue. In view of the previous, the arrivals of both types of cells to an output queue are generated according to a two-dimensional binomial process. It is fully characterized by the following joint pgf

$$
A\left(z_{1}, z_{2}\right)=\left(1-\frac{\lambda_{1}}{N}\left(1-z_{1}\right)-\frac{\lambda_{2}}{N}\left(1-z_{2}\right)\right)^{N}
$$


Obviously, the number of class- 1 and class-2 arrivals at an output queue during a slot are correlated. This is simply demonstrated by the following observation: when $m$ class- 1 cells arrive at the tagged queue during a slot $(0 \leq m \leq N)$, the maximum number of class-2 arrivals during the same slot is limited by $N-m$. We note that for $N$ going to infinity, the above expression becomes a product of two generating functions of Poisson distributions with means $\lambda_{1}$ and $\lambda_{2}$ respectively, and as a result, the arrival process becomes uncorrelated for both classes. In the following, we will investigate the effect of priority scheduling on some performance measures, such as mean value and variance of system contents and cell delay. We will, when possible, compare with a FIFO scheduling discipline to show the advantages and disadvantages of a priority scheduling discipline. In the remaining of this section, we assume a $16 \times 16$ switch $(N=16)$. We define $\alpha$ as the fraction of class-1 arrivals in the overall traffic mix (i.e., $\left.\alpha=\lambda_{1} / \lambda_{T}\right)$.

In Figures 3 and 4, mean value and variance of the system contents of class- 1 and class- 2 cells is shown as a function of the total arrival rate, when $\alpha=0.25,0.5$ and 0.75 respectively. We have also shown the mean value and variance of the system contents for $\alpha=0.5$ when a FIFO scheduling discipline is applied. These can be easily calculated because - in the special case of the arrival process characterized by (32) - the joint pgf of the system contents of both classes is given by $U_{T}\left(\alpha z_{1}+(1-\alpha) z_{2}\right)$ when a FIFO scheduling discipline is applied. One can easily see the influence of priority scheduling: the mean, as well as the variance of the number of class- 1 cells in the system is severely reduced by the HOL priority scheduling; the opposite holds for class-2 cells. In addition, it also becomes apparent that increasing the fraction of high priority cells in the overall mix increases the amount of high priority traffic while decreasing the amount of low priority traffic in the buffer. Finally, it is also clear that the impact of priority scheduling is more important if the load is high.

[Figure 3 about here.]

[Figure 4 about here.]

In Figure 5, the correlation coefficient $\rho_{u_{1} u_{2}}$, which quantifies the correlation between the number of class-1 and class- 2 cells in the system during a slot, is shown as a function of the total 
arrival rate for $\alpha=0.25,0.5$ and 0.75 . We see that $\rho_{u_{1} u_{2}}$ increases when the fraction of class- 1 cells increases (for a given total load). This can easily be understood by the priority scheduling. The influence of class- 1 cells on class- 2 cells will become more important, when the fraction of class- 1 cells increases. A second observation is that $\rho_{u_{1} u_{2}}$ is (slightly) negative when the total load is small, but becomes positive when the total load is large. The reason for this are two counteracting mechanisms. The first one is the switch structure. When more class-1 cells arrive at the switch, there will be less class-2 cells arriving at the same time (since the amount of inlets is limited), and vice versa. This negative correlation between cell arrivals of the two priority classes during a slot shows for small values of $\lambda_{T}$. For these parameter values, there is virtually no queueing and the buffer behavior is mainly determined by the number of arrivals during a single slot. The second influence is priority scheduling. As $\lambda_{T}$ (and $\lambda_{1}$ ) further increases, more and more cells are being queued, and the presence of high priority cells starts to seriously hinder the transmission of low priority cells, thereby leading to a positive correlation between $u_{1}$ and $u_{2}$. Finally, when $\lambda_{T}$ approaches 1, the total system contents (and the number of class-2 cells) approaches infinity, due to the system becoming unstable. As a result $\rho_{u_{1} u_{2}}$ approaches 0 . We have also shown the correlation coefficient for $\alpha=0.5$, when a FIFO scheduling is applied. We see that the correlation coefficient in this case is always larger than when a priority scheduling discipline is applied. Since the system contents of both classes becomes infinite when $\lambda_{T}$ approaches $1, \rho_{u_{1} u_{2}}$ approaches 1 .

[Figure 5 about here.]

Figures 6 and 7 show the mean value and the variance of the cell delay as a function of the total load for $\alpha=0.25,0.5$ and 0.75 . In order to compare with FIFO scheduling, we have also shown the mean value and variance of the cell delay in that case. The cell delay is then of course the same for class- 1 and class- 2 cells (independent of $\alpha$ ), and can thus be calculated as if there is only one class arriving according to an arrival process with pgf $A(z, z)$. This has already been analyzed, e.g., in [5] for the special case of a multiserver output-queueing switch. We observe that the influence of HOL priority scheduling is quite large. Mean delay and delay-jitter of class-1 cells reduces considerably compared to FIFO scheduling. The price to pay is of course a bigger mean 
delay and delay-jitter for class-2 cells. If this kind of traffic is not delay-sensitive, this is not too big a problem. Nevertheless, in a buffer with limited storage, an appropriate Loss priority scheme will have to be applied in order to avoid excessive cell loss of class-2 cells. Also note that it follows from these figures that increasing the fraction of high priority cells in the overall traffic mix, increases the delay of high and low priority cells.

[Figure 6 about here.]

[Figure 7 about here.]

We have shown in section 7 , that the tails of class- 2 system contents and cell delay can have 3 types of behavior, depending on which singularity of the respective pgf's is dominant. In case of the output queueing switch considered in this section, Figure 8 shows for which combination of class-1 and class-2 arrival rates the transition type behavior occurs for the system contents and cell delay, i.e., for which combination of arrival rates the regular pole and the branch point coincide. Above the curves, the tail behavior is geometric, while below the curves the tail behavior is typically non-geometric. Note that in the area above the linear line (defined by $\left.\lambda_{1}+\lambda_{2}=1\right)$ in Figure 8, the total load is larger than 1 , and as a result, the system becomes unstable.

[Figure 8 about here.]

Figures 9 and 10 show the tail behavior of the system contents and cell delay of class-1 and class-2 cells if $\lambda_{1}=0.4$ and $\lambda_{2}=0.1$ (non-geometric behavior), approximately 0.21 (transition type behavior) and 0.4 (geometric behavior) respectively. Tail behavior of system contents and cell delay of class-1 cells is of course the same for the three cases, since the arrival process of class-1 cells does not change. We have our approximations also compared with simulation results (marks in the figures). The figures show that the approximations for the class-1, the geometric and transition type tail behavior of system contents and delay is very good in these cases. The approximations of the tails of the non-geometric case are not as good, but still satisfactory. The approximations of the tails are not good in all cases though, as is shown in Figures 11 and 12. In these Figures, the tail probabilities of the system contents and cell delay of class- 2 cells is 
shown, with the parameters of the transition type behavior of the previous examples $\left(\lambda_{1}=0.4\right.$ and $\lambda_{2}=0.21$. We have shown the three types of behavior, i.e., the tail behavior just before you have the transition from non-geometrical to geometrical tail behavior, the transition type tail behavior itself, and the tail behavior just after the transition. These tail probabilities should be very near to each other, but the Figures show this is not the case. The incorrectness of the geometrical and nongeometrical approximations is due to the single-pole approximations. If both singularities lie near to each other, which is the case near the transition from non-geometric to geometric behavior, a single-pole approximation is clearly not good enough. More accurate approximations are necessary in those cases, but this lies outside the scope of this paper.

[Figure 9 about here.]

[Figure 10 about here.]

[Figure 11 about here.]

[Figure 12 about here.]

To conclude this section, we analyse the following case-study. Consider two traffic classes generating cells that arrive in a common multiplexer buffer where they are temporarily stored before transmission. The cell arrival process of both classes is described by a joint pgf given by expression (32). For both classes, their respective cell delay must satisfy the constraint $\operatorname{Prob}\left[d_{j}>T_{j}\right]<10^{-X_{j}}$, i.e., the fraction of cells of class- $j$ that have a delay larger than the treshold $T_{j}$ may not exceed $10^{-X_{j}}$, where $T_{j}$ and $X_{j}$ depend on the application under consideration. It is assumed that class-1 cells are delay-sensitive, implying that they are given priority over class-2 packets (and $T_{1}<T_{2}$, since it makes no sense to have a higher delay treshold for delay-sensitive traffic). Class-2 traffic may be loss-sensitive, and the amount of packets that is rejected due to a delay treshold being exceeded must be sufficiently small. Therefore, in the remainder we will set $X_{2}=9$ and $X_{1} \equiv X$ (where the latter may be varied). It is clear that the performance of both traffic classes, in particular their delay characteristics, can be studied using the results derived throughout this paper. 
The question we wish to answer is the following: what is the maximal load (denoted by $\rho_{T, \max }$ ), as a function of the traffic mix $\alpha$, that still fulfils the two constraints? In Figure 13, we show the maximal load as a funtion of $\alpha$ when $T_{1}=10, T_{2}=100$ and $X=1, \ldots, 9$. The constraint for the delay of class- 2 cells is the same for all $X$, i.e., Prob $\left[d_{2}>100\right]<10^{-9}$. For $X<6$, we see that this constraint is the decisive one. We notice that the maximal load suddenly lowers a reasonable amount when $\alpha$ reaches approximately 0.7 . At this point, the tail behavior changes from geometric to non-geometric tail behavior. The sudden change near 0.7 is probably due to the lack of accurateness in the tail behavior of the class-2 delay near the transition (as discussed earlier). Near this value for $\alpha$, the maximal load we find is thus not that accurate, but one can see that the incorrectness is in the order of a few percentages. For higher $X$, the constraint for the delay of the high-priority traffic becomes decisive for high $\alpha$, i.e. when more class-1 cells arrive. In Figure 14, we show $\rho_{T, \max }$ as a funtion of $\alpha$ when $X=4, T_{2}=100$ and $T_{1} \geq 3$. The constraint for the delay of class- 2 cells is again the same for all $T_{1}$. For $T_{1}>7$, we see that this constraint is the decisive one. For lower $T_{1}$, the constraint for the delay of the high priority traffic becomes decisive for high $\alpha$, i.e. when more class-1 cells arrive. Finally, in Figure 15, the maximum load as a function of $\alpha$ is shown, when $X=4, T_{1}=10$ and several values of $T_{2}$. For low $T_{2}$, the constraint for the low priority traffic is always the most stringent, while for $T_{2}>150$, the constraint for the high priority traffic is decisive for high $\alpha$. The behavior depicted in these three figures can be explained as follows. For $\alpha=0$, the traffic mix consists of low-priority packets only, and $\rho_{T, \max }$ is relatively high, depending on the value of $T_{2}$. As $\alpha$ increases, $\rho_{T, \text { max }}$ gradually decreases (but is still determined by $T_{2}$ ) since the growing fraction of high-priority packets causes the mean lowpriority packet delay to rise. Then, as $\alpha$ further increases, a transition point is reached, which is defined as the value of $\alpha$ and $\rho_{T}$ for which $\operatorname{Prob}\left[d_{1}>T_{1}\right]=10^{-X}$ and $\operatorname{Prob}\left[d_{2}>T_{2}\right]=10^{-9}$. Beyond this transition point, the bounding set by $T_{1}$ becomes predominant, and $\rho_{T, \max }$ further decreases due to the ever increasing presence of high-priority packets in the traffic mix. These figures show that the maximum allowable load can strongly depend on the delay boundaries $T_{1}$ and $T_{2}$ set on the high- and low-priority packet delays, and the traffic mix $\alpha$. 
[Figure 13 about here.]

[Figure 14 about here.]

[Figure 15 about here.]

\section{Conclusions}

In this paper, we analyzed a queueing system with a HOL priority scheduling discipline. A generating-functions-approach was adopted, which led to closed-form expressions of performance measures, such as mean and variance of the system contents and cell delay, and the correlation coefficient of the system contents of both types of cells, that are easy to evaluate. Furthermore, the tail behavior of system contents and cell delay is studied. We have shown that non-geometric tails can occur for system contents and cell delay of the low priority traffic. The model included possible correlation between the number of arrivals of the two cell types during a slot. Therefore, the results could be used to evaluate the performance of a prioritized output-queueing switch with Bernoulli arrivals.

\section{Acknowledgements}

The authors like to thank the anonymous referees for their constructive suggestions which led to the improvement of this paper.

\section{Appendix 1 : Calculation of the Probability Mass Function}

Given a generating function $X(z) \triangleq \sum_{n=0}^{\infty} x(n) z^{n}$, the question is how to find an explicit, practically usable expression for its corresponding $\operatorname{pmf} x(n)$. From the definition of $X(z)$ it follows that $x(n)$ is the coefficient of $z^{n}$ in the expansion of $X(z)$ about $z=0$, or equivalently the coefficient of $z^{-1}$ in the expansion of $z^{-1-n} X(z)$ about $z=0 . x(n)$ is thus by definition the residue of the function $z^{-1-n} X(z)$ in the point $z=0$. Since $z=0$ is an $n$-multiple pole of $z^{-1-n} X(z)$, calculating 
the residue in $z=0$ is nearly impossible for large $n$ (since evaluating the residue in an $n$-multiple pole requires $n$ derivations). Using the residue theorem of Cauchy however, it is proven that

$$
\begin{aligned}
x(n) & =\operatorname{Res}_{z=0}\left[X(z) z^{-1-n}\right] \\
& =\frac{1}{2 \pi i} \oint_{C_{1}} X(z) z^{-1-n} d z-\sum_{j=0}^{m} \operatorname{Res}_{z=z_{j}} X(z) z^{-1-n}
\end{aligned}
$$

with $i=\sqrt{-1}, C_{1}$ a contour with infinite radius and $z_{j}$ the poles of $X(z)$. The contour integral in the former expression is normally easy to calculate (in most cases the term equals zero). If we are only interested in the expression of $x(n)$ for large $n$, the sum of residues can be approximated by the residue in the dominant pole of $X(z)$ (the approximation is exact for $n \rightarrow \infty$ ). As a result, an easy, practically usable formula to calculate approximate tail probabilities is obtained.

\section{Appendix 2 : Inversion of $(1-z)^{\alpha}$}

Theorem 1 Assume that, with the sole exception of the singularity $z=1$,

$$
F(z) \triangleq \sum_{n=1}^{\infty} f(n) z^{n}
$$

is analytic in the domain

$$
\Delta=\{z:|z| \leq 1+\eta,|\operatorname{Arg}(z-1)| \geq \theta\}
$$

in which $\eta$ is a positive real number and $0<\theta<\pi / 2$. Assume further that as $z$ tends to 1 in $\Delta$,

$$
F(z)=K(1-z)^{\alpha},
$$

with $\alpha \notin \mathbb{N}$. Then, as $n \rightarrow \infty$,

$$
f(n)=\frac{K}{\Gamma(-\alpha)} n^{-\alpha-1}
$$




\section{References}

[1] Bae JJ, Suda T. Survey of traffic control schemes and protocols in ATM networks. Proceedings of the IEEE 1991; 79(2):170-189.

[2] Bruneel H. Analysis of buffer behaviour for an integrated voice-data system. Electronics Letters $1983 ; 19(2): 72-74$.

[3] Bruneel H, Kim BG. Discrete-time models for communication systems including ATM. Kluwer Academic Publishers, Boston, 1993.

[4] Bruneel H, Steyaert B. Buffer requirements for ATM switches with multiserver output queues. Electronics Letters 1991;27(8):671-672.

[5] Bruneel H, Steyaert B, Desmet E, Petit GH. An analytical technique for the derivation of the delay performance of ATM switches with multiserver output queues. International Journal of Digital and Analog Communication Systems 1992;5:193-201.

[6] Bruneel H, Steyaert B, Desmet E, Petit GH. Analytic derivation of tail probabilities for queue lengths and waiting times in ATM multiserver queues. European Journal of Operational Research 1994;76(3):563-572.

[7] Drmota M. Systems of functional equations. Random Structures \& Algorithms 1997;10(12):103-124.

[8] Flajolet P, Odlyzko A. Singularity analysis of generating functions. SIAM Journal on discrete mathematics 1990;3(2):216-240.

[9] Hluchyj MG, Karol MJ. Queueing in high-performance packet switching. IEEE Journal on Selected Areas in Communications 1988;6(9):1587-1597.

[10] Khamisy A, Sidi M. Discrete-time priority queues with two-state Markov Modulated arrivals. Stochastic Models 1992;8(2):337-357. 
[11] Liu KY, Petr DW, Frost VS, Zhu HB, Braun C, Edwards WL. Design and analysis of a bandwidth management framework for ATM-based broadband ISDN. IEEE Communications Magazine 1997;35(5):138-145.

[12] Rubin I, Tsai ZH. Message delay analysis of multiclass priority TDMA, FDMA, and discretetime queueing systems. IEEE Transactions on Information Theory 1989;35(3):637-647.

[13] Sidi M, Segall A. Structured priority queueing systems with applications to packet-radio networks. Performance Evaluation 1983;3(4):265-275.

[14] Stanford DA. Interdeparture-time distributions in the non-preemptive priority $\sum M_{i} / G_{i} / 1$ queue. Performance Evaluation 1991;12(1):43-60.

[15] Sugahara A, Takine T, Takahashi Y, Hasegawa T. Analysis of a nonpreemptive priority queue with SPP arrivals of high class. Performance Evaluation 1995;21(3):215-238.

[16] Takahashi Y, Hashida O. Delay analysis of discrete-time priority queue with structured inputs. Queueing Systems 1991;8(2):149-164.

[17] Takine T, Sengupta B, Hasegawa T. An analysis of a discrete-time queue for broadband ISDN with priorities among traffic classes. IEEE Transactions on Communications 1994;42(2-4):18371845.

[18] Takine T. A nonpreemptive priority MAP/G/1 queue with two classes of customers. Journal of Operations Research Society of Japan 1996;39(2):266-290.

[19] Van Mieghem P, Steyaert B, Petit GH. Performance of cell loss priority management schemes in a single server queue. International Journal of Communication Systems 1997;10(4):161-180.

[20] Xiong Y, Bruneel H. Buffer contents and delay for statistical multiplexers with fixed-length packet-train arrivals. Performance Evaluation 1993;17(1):31-42. 


\section{List of Figures}

1 The queueing system . . . . . . . . . . . . . . . . . . . . . . . . . 29

2 An $\mathrm{NxN}$ output queueing switch . . . . . . . . . . . . . . . 30

3 Mean value of system contents versus the total arrival rate . . . . . . . . . . . . 31

4 Variance of system contents versus the total arrival rate . . . . . . . . . . . . 32

5 Correlation of system contents versus the total arrival rate . . . . . . . . . . . . 33

6 Mean value of cell delays versus the total arrival rate . . . . . . . . . . . . . . . 34

$7 \quad$ Variance of cell delays versus the total arrival rate . . . . . . . . . . . . . . 35

8 Regions for tail behavior as a function of the arrival rates of both classes . . . . . . 36

9 Tail behavior of the high and low priority system contents for some combinations of class-1 and class-2 arrival rates . . . . . . . . . . . . . . . . . 37

10 Tail behavior of the high and low priority cell delay for some combinations of class-1 and class-2 arrival rates . . . . . . . . . . . . . . . . . . . 38

11 Tail behavior of the low priority system contents near the transition from nongeometrical to geometrical . . . . . . . . . . . . . . . . . 39

12 Tail behavior of the low priority cell delay near the transition from non-geometrical to geometrical . . . . . . . . . . . . . . . . . . . . . 4 40

13 Maximum load versus the fraction of class- 1 arrivals for several values of $X \ldots$. . . 41

14 Maximum load versus the fraction of class- 1 arrivals for several values of $T_{1} \ldots \ldots 42$

15 Maximum load versus the fraction of class- 1 arrivals for several values of $T_{2} \ldots 43$ 


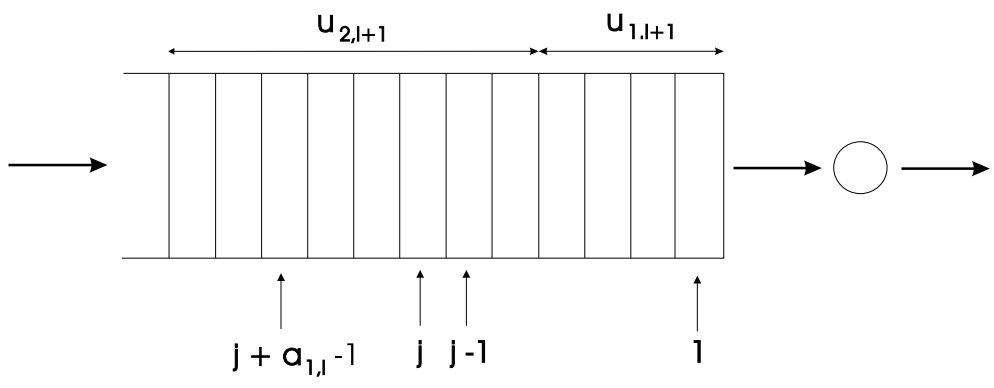

Figure 1: The queueing system 


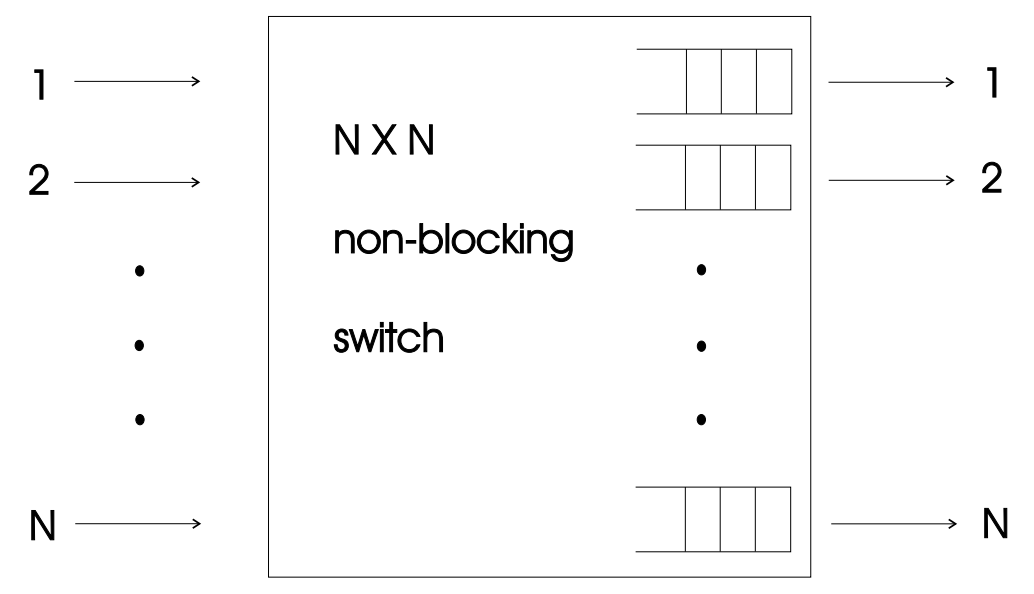

Figure 2: An NxN output queueing switch 


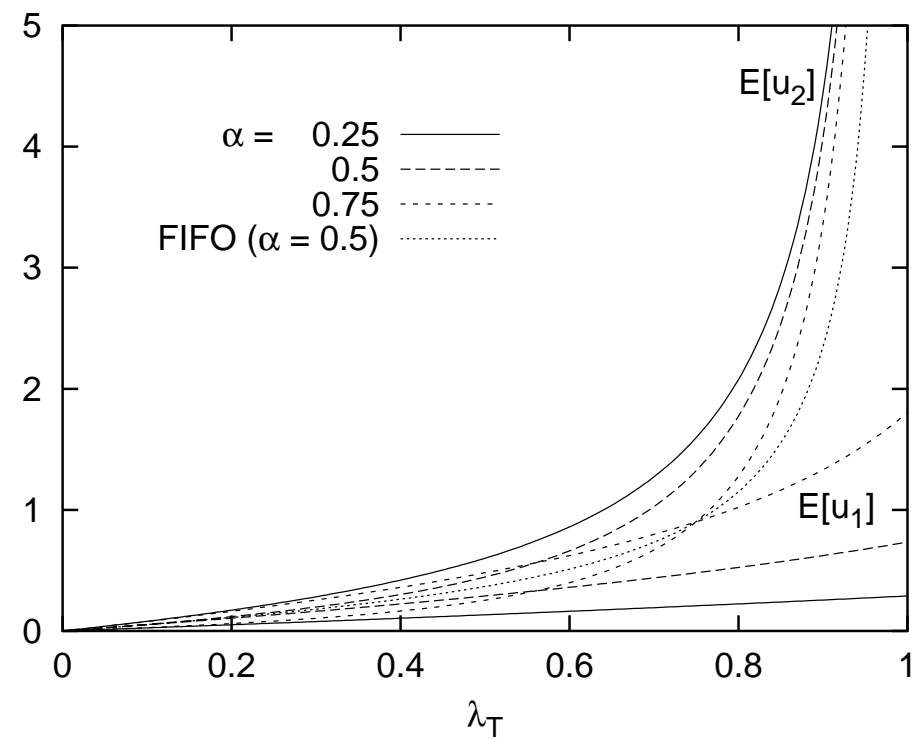

Figure 3: Mean value of system contents versus the total arrival rate 


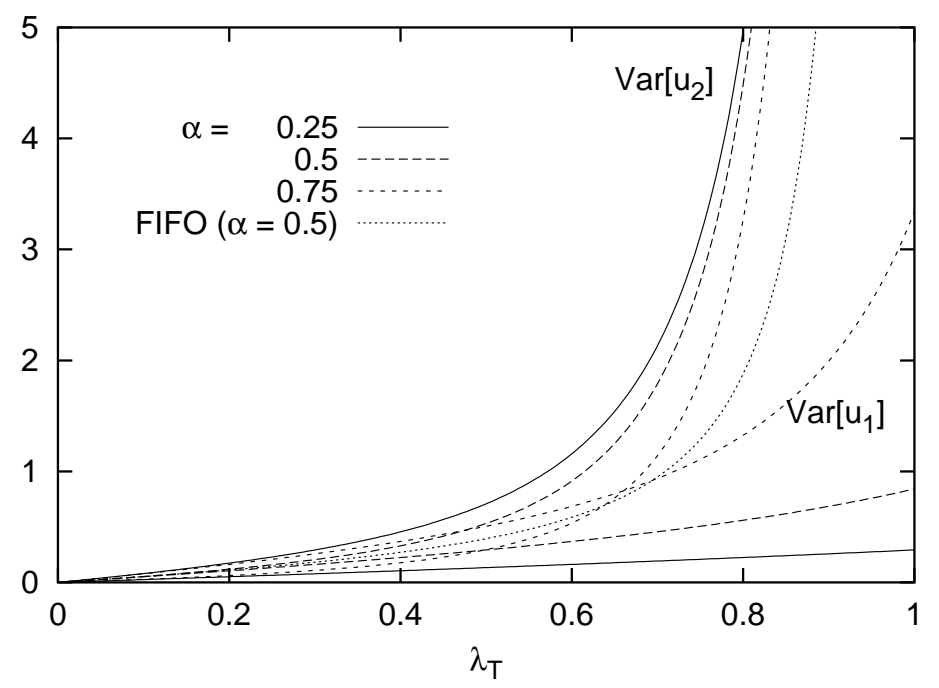

Figure 4: Variance of system contents versus the total arrival rate 


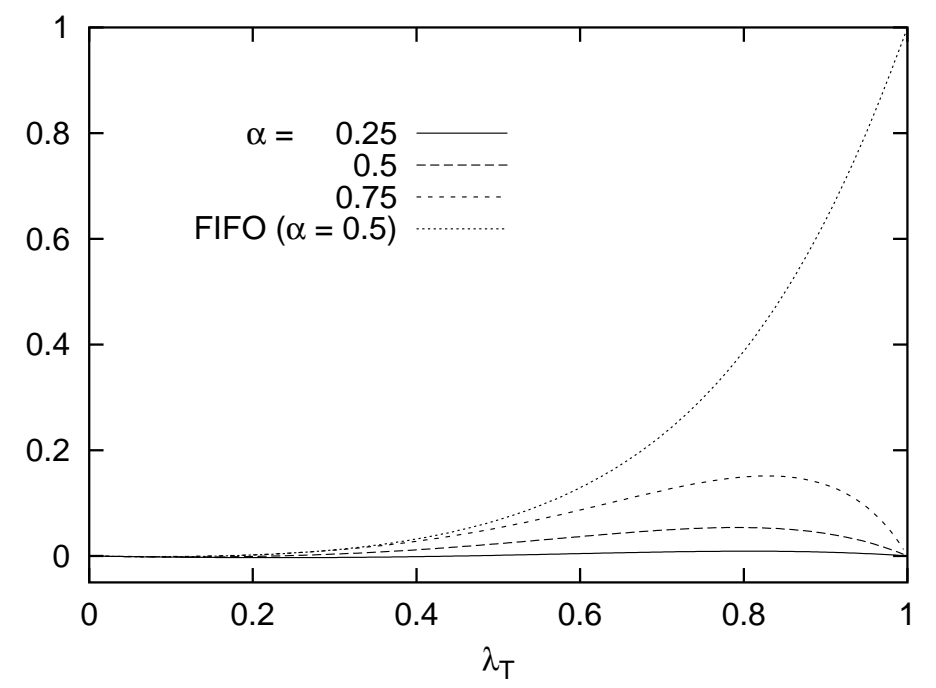

Figure 5: Correlation of system contents versus the total arrival rate 


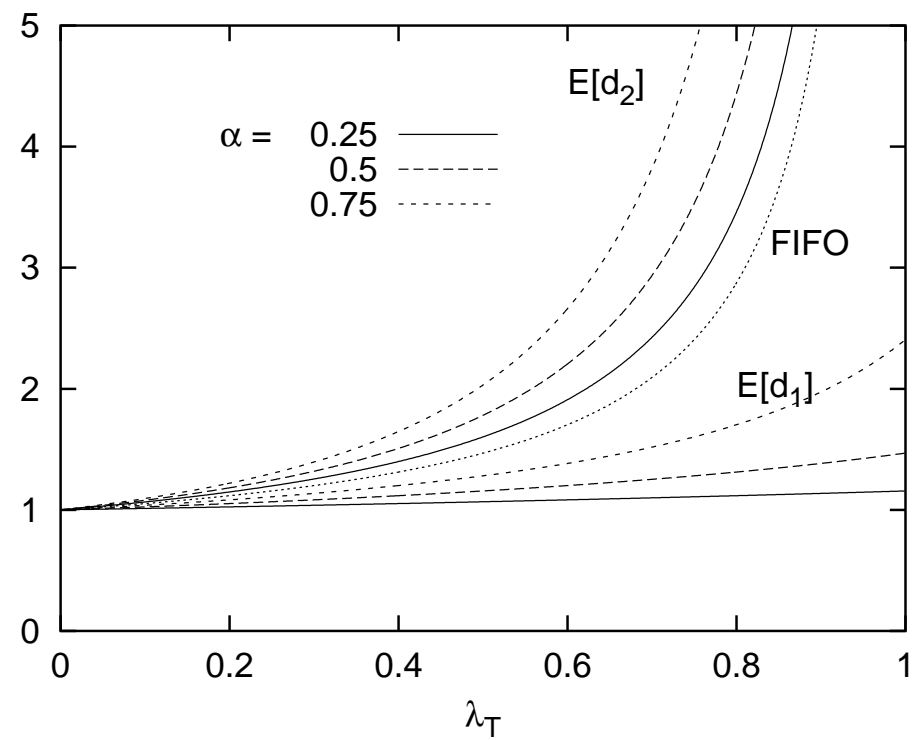

Figure 6: Mean value of cell delays versus the total arrival rate 


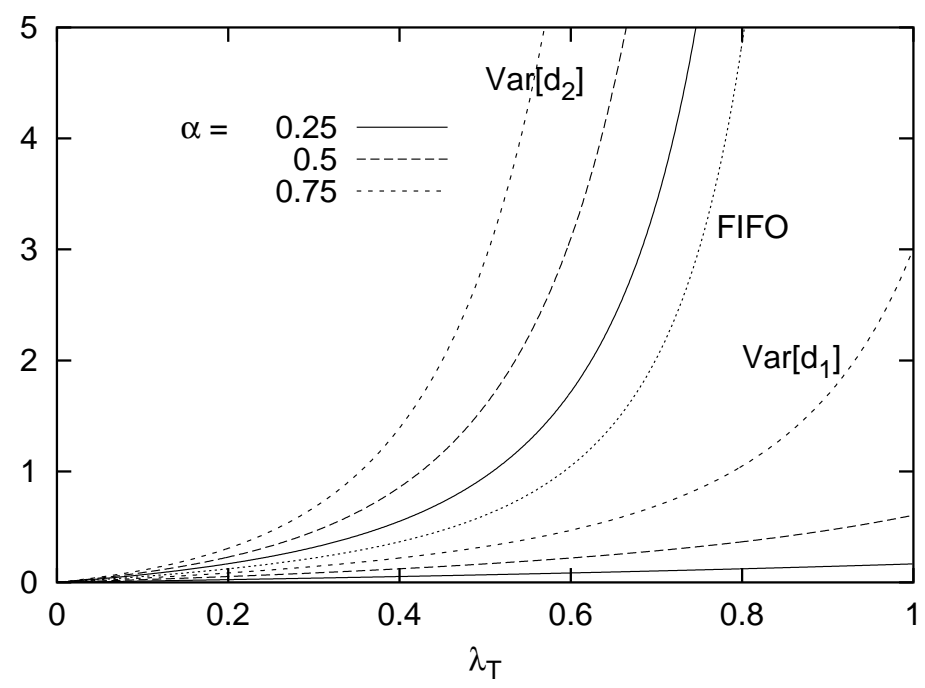

Figure 7: Variance of cell delays versus the total arrival rate 


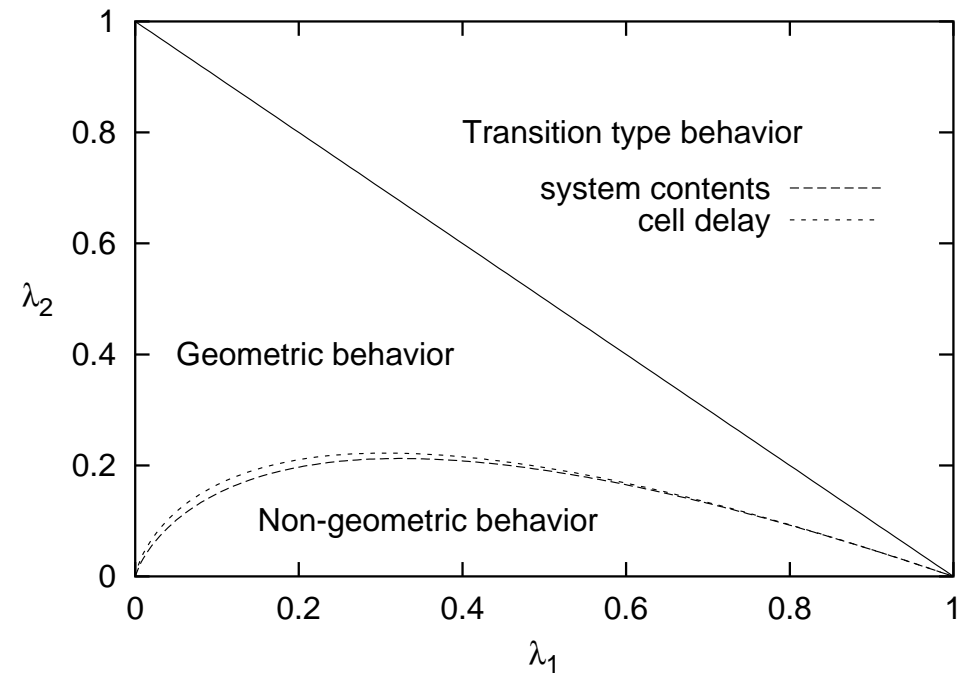

Figure 8: Regions for tail behavior as a function of the arrival rates of both classes 


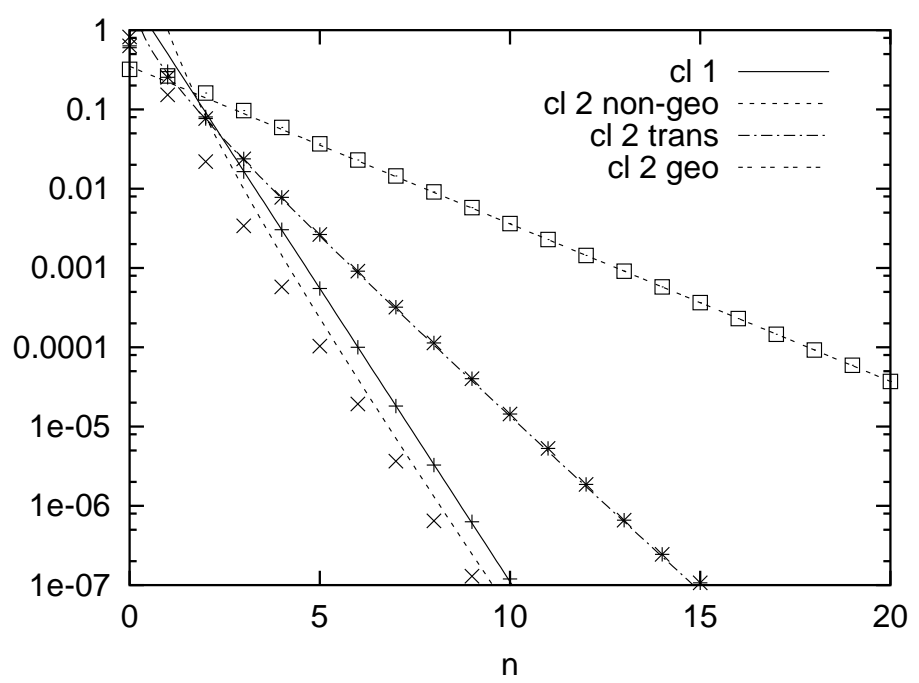

Figure 9: Tail behavior of the high and low priority system contents for some combinations of class- 1 and class- 2 arrival rates 


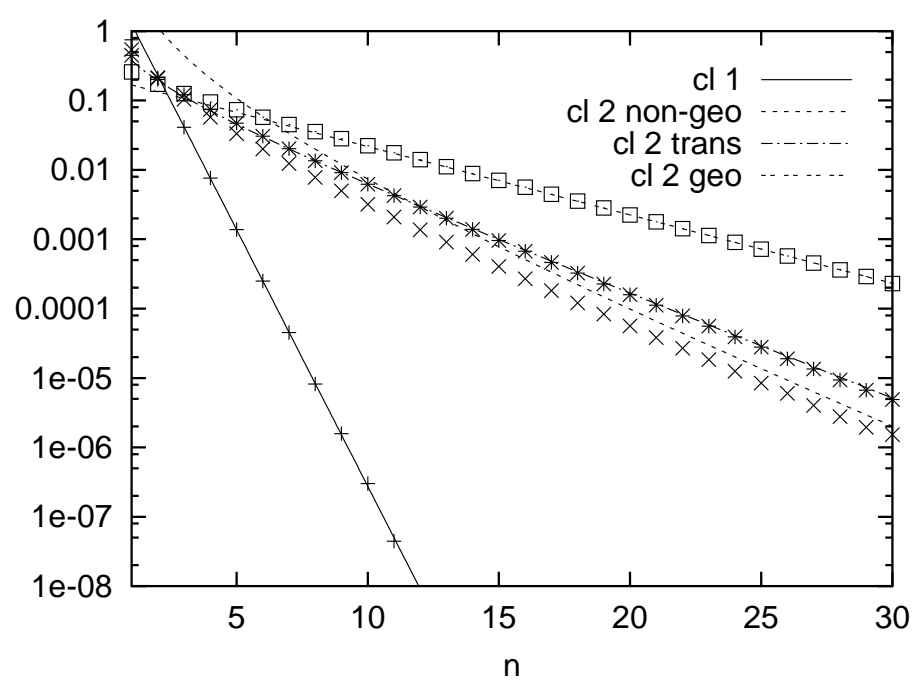

Figure 10: Tail behavior of the high and low priority cell delay for some combinations of class-1 and class- 2 arrival rates 


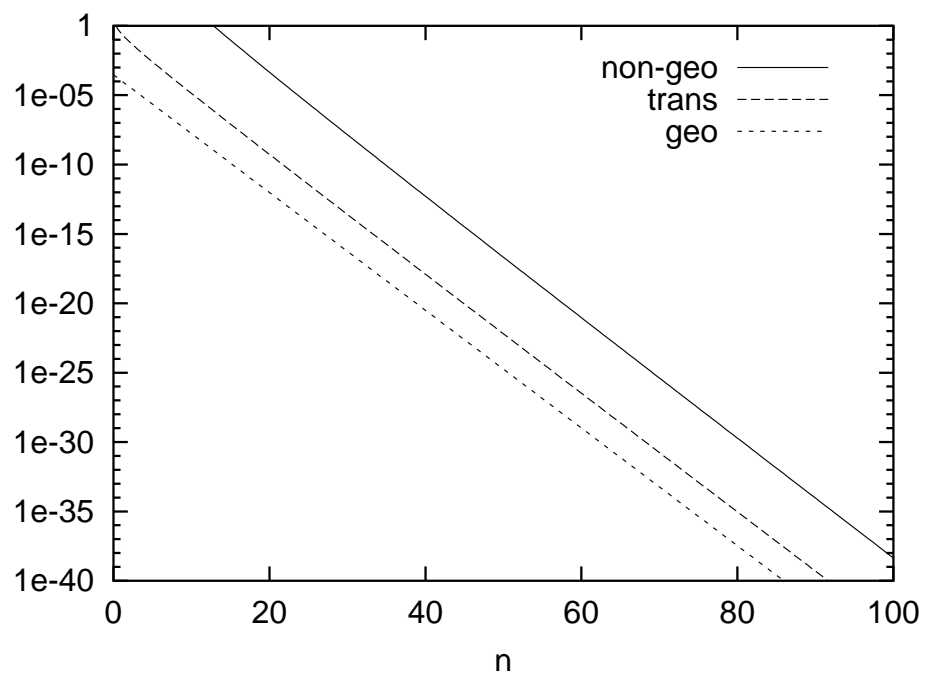

Figure 11: Tail behavior of the low priority system contents near the transition from nongeometrical to geometrical 


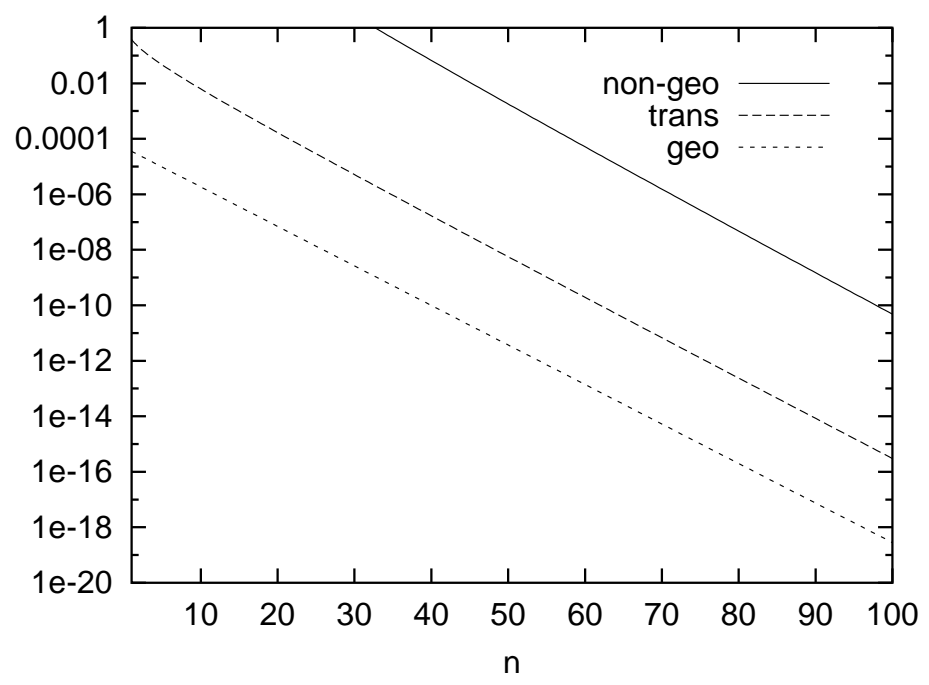

Figure 12: Tail behavior of the low priority cell delay near the transition from non-geometrical to geometrical 


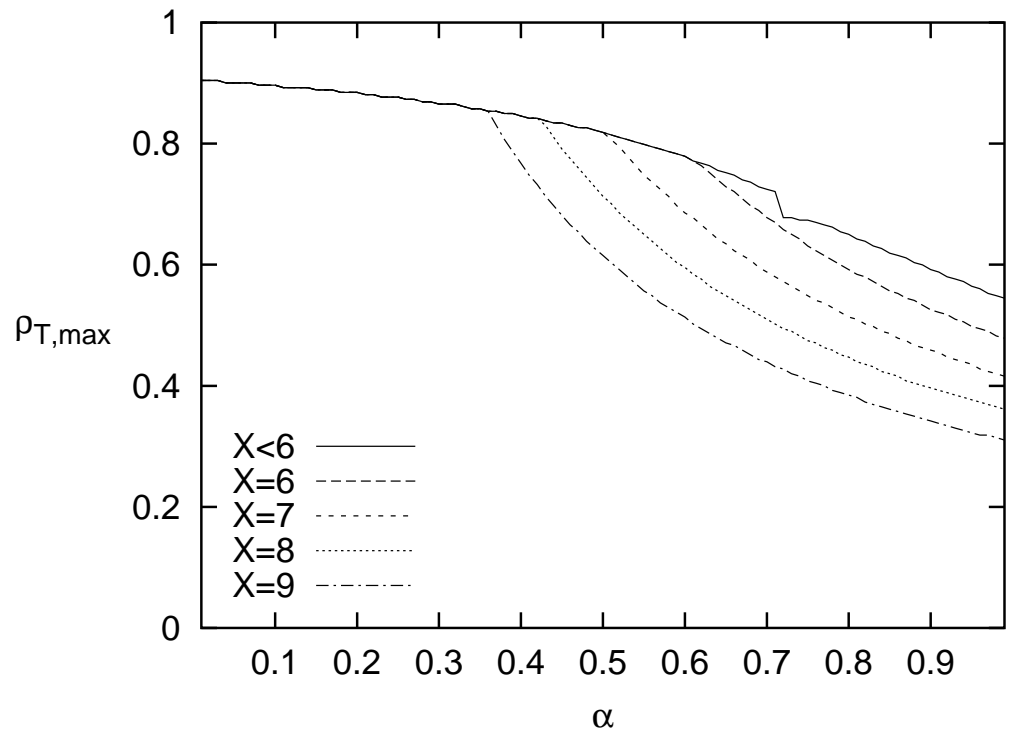

Figure 13: Maximum load versus the fraction of class-1 arrivals for several values of $X$ 


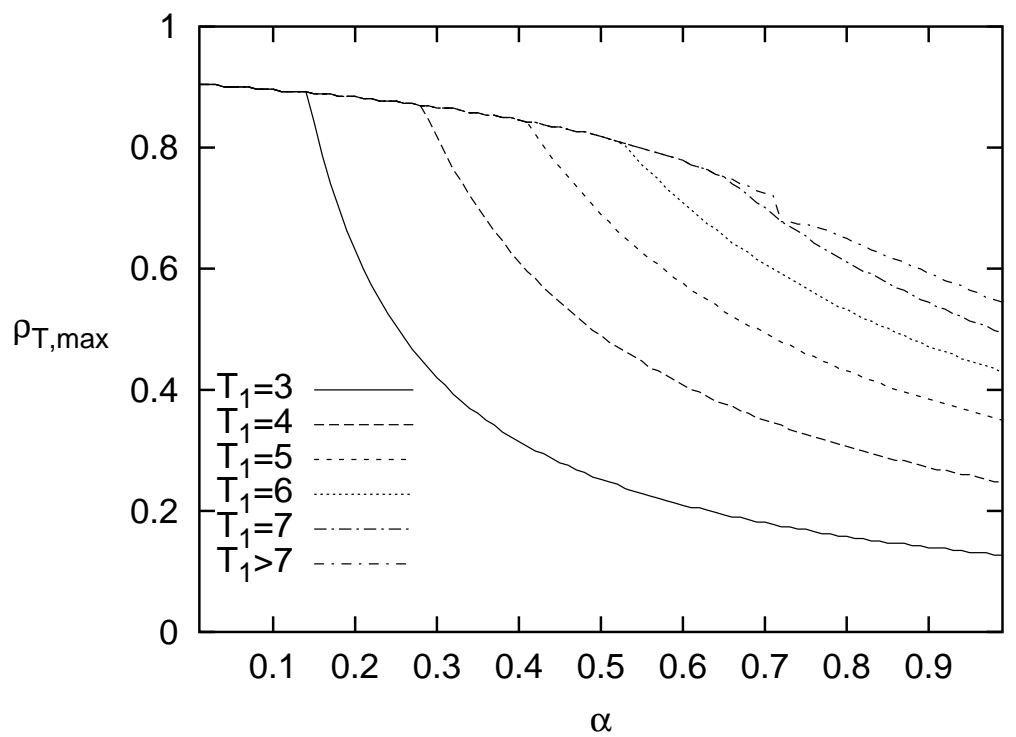

Figure 14: Maximum load versus the fraction of class-1 arrivals for several values of $T_{1}$ 


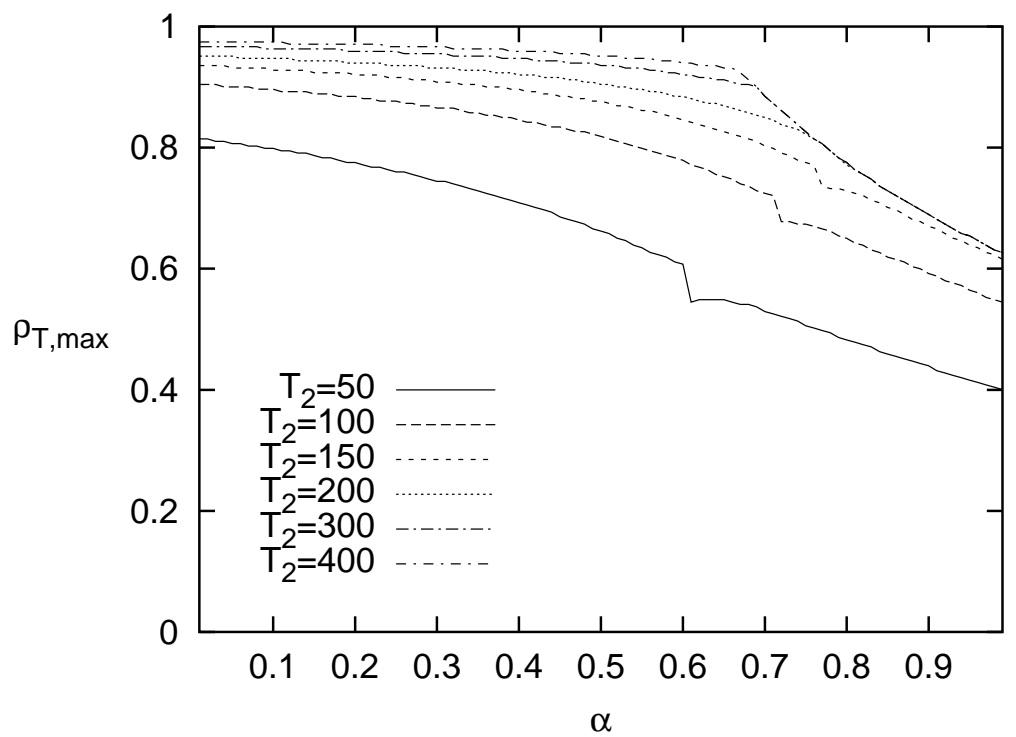

Figure 15: Maximum load versus the fraction of class-1 arrivals for several values of $T_{2}$ 